\title{
Modeling simulation and performance evaluation of low voltage power line communication channel
}

\author{
Zahoor Alam $^{1}$, Aizad Khursheed ${ }^{2 *}$ and Rahul Kant Chaudhary ${ }^{3}$ \\ Industrial Trainer, Amity Education Services Pvt Ltd., Bikaner, Rajasthan, India ${ }^{1}$ \\ Assistant Professor, Amity University, Greater Noida, India ${ }^{2}$ \\ Assistant Professor, Jamia Millia Islamia, New Delhi, India ${ }^{3}$
}

\section{(C)2018 ACCENTS}

\begin{abstract}
Power line carrier communication (PLCC) is a communication technology used for transmitting information using power lines as the communication media. PLCC techniques have been used either by electrical utilities for control and monitoring of their distribution networks, or for simple home automation systems. With rapid growth in home networks, PLCC methods are a flexible way to implement low cost, reliable and widely accessible networks in the domestic environment. The major benefit of power line communication is the utilization of an existing infrastructure.
\end{abstract}

\section{Keywords}

Power line communication, Line parameters, Matlab simulator, Channel model, Transfer function.

\section{Introduction}

Power line communication is a type of communication method which utilize existing infrastructure that is known with different name that are power line networking, mains communication, power line telecom or power band. It was originally proposed in the early 1800 's; in 1838 the first remote electricity supply metering was proposed. The power line was initially designed to distribute power in an efficient way. The first patents on power line signalling were proposed in the UK in 1897 and the technology used as early as the 1920's by utilities such as the electric power utility of London. Early use of the power line communication technology was to remotely control some of its equipment including circuit breakers and high voltage switches. This application of the technology only required the transfer of data at relatively at low speeds of (less than $30 \mathrm{kbps}$ ) transfer rate and is still in use by several utilities today [1].

The basic principle behind power line communication is that low-frequency $50 \mathrm{Hertz}(\mathrm{Hz})$ or $60 \mathrm{~Hz}$ portion is used for power flow and unused space that is higher frequency portion for data flow, as shown in Figurel.

*Author for correspondence

308

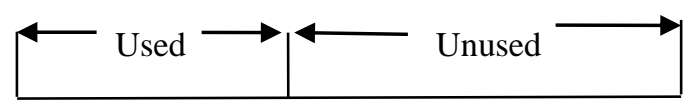

$50 \mathrm{~Hz}$ frequency

Figure 1 Power line communication

The communication signal is transmitted from one point to another through a physical path called communication channel. Power-line communication is basically propagation of information carrying electrical signals over power-line to be utilized as a channel. In power line carrier communication, a high frequency signal is superimposed on $50 \mathrm{~Hz}$ or $60 \mathrm{~Hz}$ electrical signal. Electrical power lines are classified as high voltage that is greater than $100 \mathrm{KV}$, medium voltage that is between $1 \mathrm{KV}$ to $100 \mathrm{KV}$ and low voltage that is less than $1 \mathrm{KV}$ in voltage network. The idea is to carry out transmission at low energy levels. The high frequency signal is conveyed, acknowledged and decoded by means of the power infrastructure. Recent research is mainly focused on increasing the bit rate to support high-speed network applications [2].

The objective of this paper is to do modeling of low voltage power line communication channel using different types of line parameters for the feasibility of line technologies for different applications in an effective manner to develop a simplified transfer characteristic model for the low voltage channel for power line communication based on the two-wire 
transmission line theory using mat lab simulator and to check performance of that simulator. A bottom-up approach based on the frequency-domain modeling using scattering matrix is implemented for channel modeling in this paper.

PLCC channel models can be grouped into physical (bottom-up) and parametric (top-down) models. The physical models describe the electrical properties of a transmission line, for example, through the specification of cable type (line parameters), the cable length and the position of branches while parametric models use a much higher level of notion from the physical reality and describe the channel, for example, through its impulse response or transfer function. Further, within each group, it can be distinguished between deterministic and stochastic models. While deterministic models aim at the description of one or a small set of specific reproducible PLC channel realizations, stochastic models aim at reflecting a wide range of channel realizations according to their probability of occurrence [3].

This paper is organized as follows: Introduction of power line communication is given in section 1, an overview of power line communications for low voltage, taking example of an application is discussed in section 2, and also, how power line communication can be integrated into the existing infrastructure is mentioned in this section. Section 3 deals with the different types of parameters of power line communication that are used, in different modeling approaches for simulation of transfer characteristics of power line communication channel which are discussed in section $4 \& 5$. Channel modeling, simulation of transfer characteristics and performance evaluation of channel is discussed in section 6 . Section 7 contains the concluding remarks.

\section{Low voltage power line application}

Application of PLCC technology has been experiencing significant attention due to a combination of factors such as the recent advances made in the PLC industry lead to the development of two spheres of the technology, in-home PLC and access PLC [4].

Home PLC has been in use since the 1980's when power lines were used to connect computers and peripheral such as printers and plotters within an office building socket as shown in Figure 2.

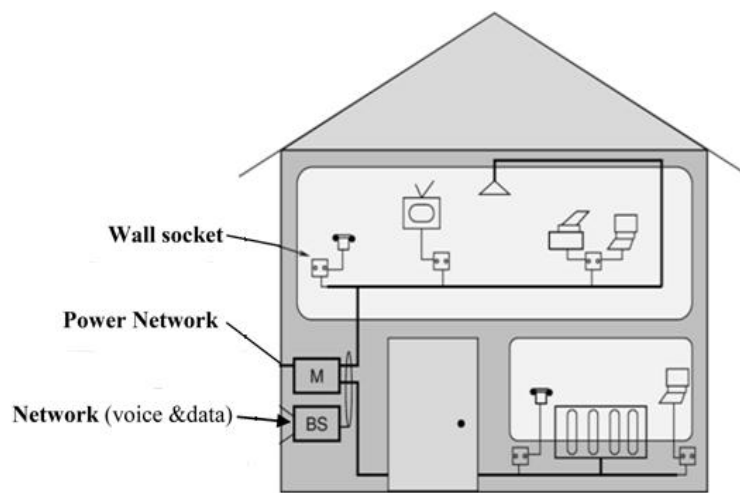

Figure 2 Home power line communication

Access PLC, as the name implies, is the power line technology geared to serve as a last mile access technology for getting communication signals to the home, much like cable and digital subscriber line (DSL), over the power distribution network. This feat is achieved by connecting the medium to low voltage distribution network to a communication backbone at the distribution transformer [10] as shown in Figure 3 .

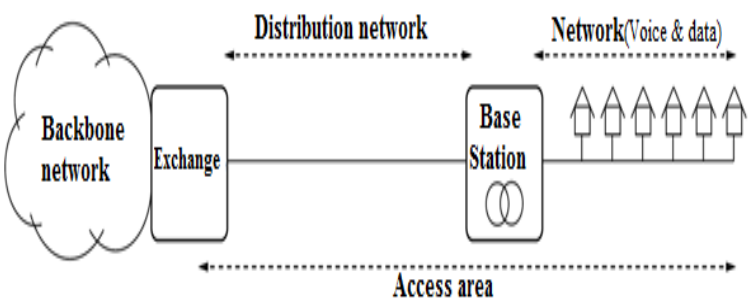

Figure 3 Access power line communication

PLCC technology is used for many applications with combination of other technologies to give chip networking solutions in low voltage power sector [4]. These are some key areas which used power line communication:

a. Automatic meter reading (AMR): PLCC is a one of the technology which transform traditional meter to smart meter that send real time data from meter.

b. Advance metering infrastructure (AMI): It is an integrated system of smart meter, communication network, and data management system that provide two-way communications between home meter and host central station.

c. Demand side management (DSM): It is used to maintain demand accordance to the supply and it may give a lot better control especially under peak power conditions and allows customer to be central part of energy management program. 
d. Protective relaying: In power sector, protection is very important area which basic objective to sense problems with power system components and isolates the components from rest of the systems.

e. Micro grid: It is local grid which integrated energy system that manages the interconnected loads and distributed energy resources using power line communication technology. The generation and distribution of power can be operated in island mode or grid connected mode.

f. Energy storage: Power line communications, both at the supply side and the demand side, would provide the necessary visibility in the grid by being able to monitor and control the amount of electricity storage in near real-time.

According to applications, PLC can be broadly viewed as: Narrowband PLC and Broadband PLC, Narrowband Power line communication has low data rates, frequency, and has longer range (up to several kilometres), which can be extended using repeaters. Broadband Power line communication has high data rates, frequencies and has shorter-range applications [5].

\section{Parameters of low voltage transmission line}

There are two types of parameters in transmission line that are primary parameters and secondary parameters. These are known as distributed parameters of transmission line that's depends on cable specification and it is approximated [3]. The power line cables are approximated to be a two-wire transmission line with solid core conductor for the ease of implementation using software simulation as shown in Figure 4.

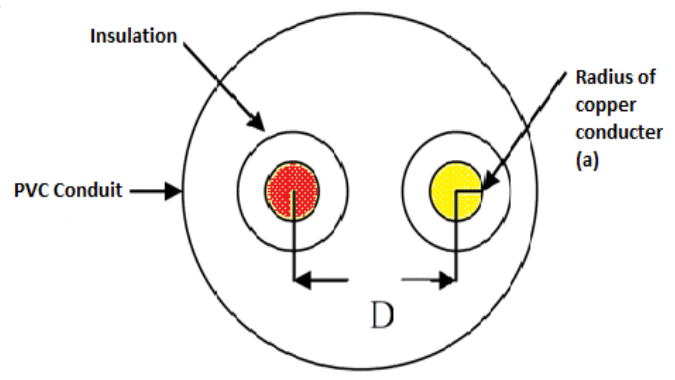

Figure 4 Approximate model of power line

The dielectric material, between the cable conductors, is in homogeneous in both space (due to the round shape of the cable conductor) and contents (mixture of insulation and air). But since the cables are in proximity to each other, the thickness of the 310 insulation " $t$ " is comparable with that of the air space between the conductors. In this model, the dielectric is assumed to be just a mixed content material and the effects of the inhomogeneous in space are neglected to keep the model tractable [6].

Here, geometrical properties and electrical properties of cable are given:

Distance between the two conductors (Live and Neutral) is $D=2 t+2 t+2 a$, where $t=$ thickness of insulation $=0.7 \mathrm{~mm}, \mathrm{a}=$ Radius of copper conductor $=0.63 \mathrm{~mm}$, therefore, distance between two conductors are $4.06 \mathrm{~mm}$, and electrical properties of cable are given be in the Table 1 below

Table 1 Electrical properties of cable

\begin{tabular}{llcc}
\hline Conductivity of copper & $\Sigma_{\mathrm{C}}$ & $5.8 \times 10^{7} \mathrm{~S} / \mathrm{M}$ \\
\hline $\begin{array}{l}\text { Relative permittivity of } \\
\text { dielectric (PVC }=4 \text { \& Air }=1)\end{array}$ & $\varepsilon_{\mathrm{r}}$ & 0.8 \\
\hline Free space permeability & $\mu_{\mathrm{g}}$ & $1.2 \times 10^{-6}(\mathrm{H} / \mathrm{m})$ \\
\hline Conductivity of dielectric & & $\sigma_{\mathrm{d}}$ & $1.0 \times 10^{-5} \mathrm{~S} / \mathrm{m}$ \\
\hline $\begin{array}{l}\text { Relative permeability } \\
\text { copper }\end{array}$ & $\mu_{\mathrm{r}}$ & 1 \\
\hline Free space permittivity & & $\varepsilon_{\mathrm{g}}$ & $8.5 \times 10^{-12}(\mathrm{~F} / \mathrm{m})$ \\
\hline
\end{tabular}

Approximated value of line is used in primary parameters that are also known as distributed parameters of transmission line [7] as shown in Figure 5.

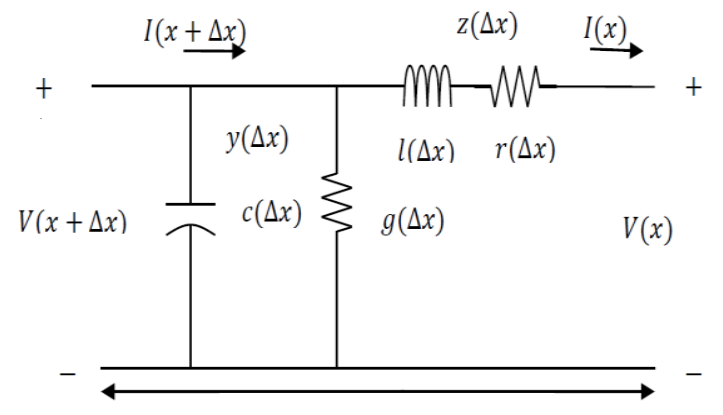

Figure 5 Equivalent circuit of two-wire transmission line

Based on the lumped-element circuit of a two-wire transmission line, the primary line parameters per unit length $(\mathrm{m})$ are:

Resistance ' $\mathrm{R}$ ' $=1 / \pi a \delta \sigma_{c}$

$\sqrt{1 / \pi f \mu_{c} \sigma_{c}}$

Where ' $\delta$ ' $=$ skin depth and is a function of frequency ' $f$ '. This effect causes an increase in the resistance of the cable and it worsens as the current frequency increases. Also, 


$$
\begin{aligned}
L & =\frac{\mu_{c}}{\pi} a \cosh (D / 2 a) \\
G & =\frac{\pi \sigma_{d}}{a \cosh (D / 2 a)} \\
C & =\frac{\pi \varepsilon_{d}}{a \cosh (D / 2 a)}
\end{aligned}
$$

Here, $\mu_{c}=$ permeability of copper conductor, $\sigma_{d}$ $=$ conductivity of the dielectric material, and $\varepsilon_{d}$ $=$ permittivity of the dielectric material.

Secondary parameters of transmission lines are characterized by their characteristic impedance $\mathrm{Z}_{\mathrm{C}}$ and their propagation constant $\gamma$. The standard formulae for characteristic impedance $Z_{C}$ and propagation constant $\gamma$ are given below in equation (5) and (6) respectively. Where $\mathrm{R}$ is the per-unit line resistance, $\mathrm{L}$ is the per-unit-inductance, $\mathrm{C}$ is the perunit-capacitance, $\mathrm{G}$ is the per-unit line conductance, and $\omega=2 \pi \mathrm{f}$. It should be noted that these basic line parameters R, L, C, and G are frequency dependent and can be obtained from expressions, given in the following sub-sections, which use the cable specifications [3].

$Z_{c}=\sqrt{\frac{z}{y}}=\sqrt{\frac{R+j w L}{G+j w C}}$
$\gamma=\sqrt{z y}=\sqrt{(R+j w L)(G+j w C)}$

Basically, there are two essential parameters in these models: the primary parameters (R, L, C and $\mathrm{G})$ and secondary parameters $\left(Z_{C}, \gamma\right)$. These two parameters determine the reliability and accuracy of the model.

\section{Modeling of power line channel}

There are two types of approaches for modeling of power line communication channel that are top down approach and bottom up approach [3]. The first approach is based on an empirical formula, it is also known as parametric approach and further divided into two approach deterministic and stochastic approach while the second is based on the transmission line theory development, it is known as physical approach and also divided into two approaches, deterministic and stochastic approach [8].

The physical (bottom-up) deterministic approach is depends on intrinsic parameters of the transmission line and connected loads in the form of ABCD or SParameters to establish a transfer function of the channel.
In this paper used scattering metrics theory to take transmission line parameters, it is also known as reflected and incident matrix theory [3].

These model parameters are discussed in section third and these parameters are used in modeling of power line channel using physical (bottom-up) deterministic approach with scattering matrix technique in frequency domain. Scattering matrix gives the relationship of the incident (a) and reflected (b) channels as shown in the Figure 6 below [9].

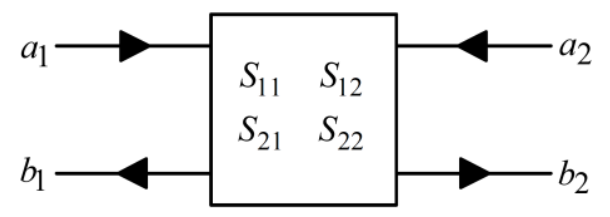

Figure 6 Two-port scattering parameters model

The S-parameter matrix for the two-port network is probably the most common and it serves as the basic building block for generating higher order matrices for larger networks [3]. In this case, relationship between the reflected, incident power channels and the $\mathrm{S}$-parameter matrix is given by:

$$
\left[\begin{array}{l}
b_{1} \\
b_{2}
\end{array}\right]=\left[\begin{array}{ll}
S_{11} & S_{12} \\
S_{21} & S_{22}
\end{array}\right]\left[\begin{array}{l}
a_{1} \\
a_{2}
\end{array}\right]
$$

The equations give the relationship between the reflected and incident power channels at each of the network ports, 1 and 2, in terms of the network's individual S-parameters $S_{11}, S_{12}, S_{21}$ and $S_{22}$, where $S_{11}$ is the input port voltage reflection coefficient, $S_{22}$ is the output port voltage reflection coefficient, $S_{12}$ is the reverse voltage gain and $S_{21}$ is the forward voltage gain and. Here, $S_{21}$ gives the network transfer function.

\section{Simulation of power line channel}

In order to find the degree of signal degradation in the power line channel between two access point, simulation is done using MATLAB to obtain the transfer function whose amplitude (dB) Vs. frequency plot gives the attenuation in the signal strength and angle (radian) Vs. frequency plot gives the phase distortion or delay. The flow cart for simulation is divided into two blocks. The first block calculates the primary line parameters of the two wire transmission lines and this transmission line parameter is used in the second block to determine the frequency response of the PLC channel [10]. Figure 7 shows the flow chart of simulation process. 


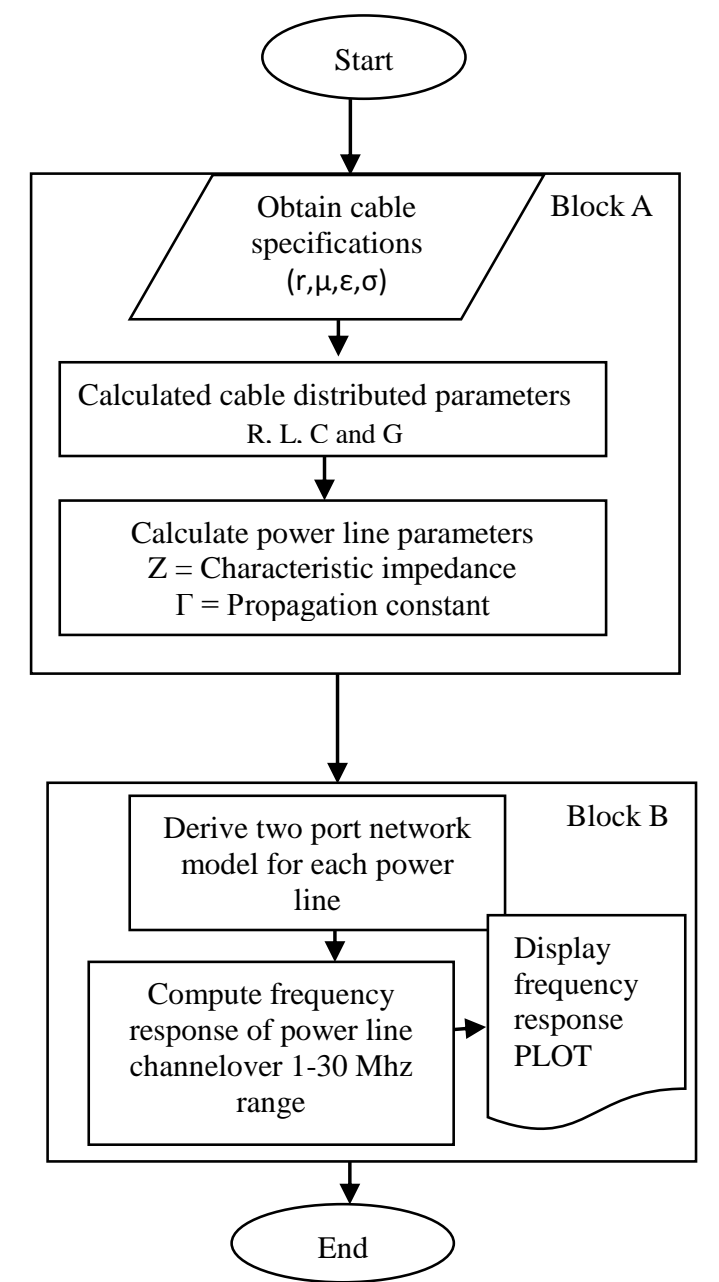

Figure 7 Flow chart of simulation process

In block $\mathrm{A}$, the distribution line parameters $\mathrm{Z}_{\mathrm{C}}$ and $\gamma$ are calculated for each individual cable size, which were selected based on the voltage level that make up the PLCC path being considered. The cable specifications (radius a, separation D, core conductor conductivity $\sigma$, insulation permittivity $\varepsilon$, and permeability $\mu$ ) from the manufacturers data sheet, for each cable size in the channel is used as input to block A. The distributed cable parameters R, L, C and $G$ are then calculated for each cable and the results used to calculate $Z_{C}$ and $\gamma$ for each distribution line segment. This data is then stored in arrays and passed to block B [10].

The inputs to block $B$ are the characteristics impedance $Z_{C}$ and propagation constant $\gamma$ for each distribution line segment. In block $\mathrm{B}$, the equivalent two-port network model for each distribution line segment is first calculated. The equivalent two-port network model representative of the complete PLCC channel path being considered is then calculated by cascading the individual Two-port network models for each segment based on the specific topology of the PLCC channel path [11]. The transfer function of the channel is then calculated and the frequency response over the $1-30 \mathrm{MHz}$ range is calculated at a $1.5 \mathrm{kHz}$ interval. Finally, the frequency response data points from block B are stored in two columns in an excel file. First column gives the frequency points used and the corresponding channel response value in $\mathrm{dB}$ is stored in the second column.

Figure 8 obtained after running the program shows the presence of deep notches at certain frequencies in the transfer function. These profound notches are outcome from signal manifestations and multipath propagation channel that create interference and delay in communication [9], for better communication between two access points, this frequency domain is not suitable. Finally concluded that, these carrier frequency and cable length will not be chosen for efficient transmission.

Also, Figure 9 illustrates that when there is profound notch at certain frequency in the power line communication channel, there is a discontinuity in the phase characteristics leading to phase distortion or delay [11].

The implementation of the analytical model developed for determining the PLC channel characteristics of the two wire transmission lines was simulated in this section using Mat lab code to determine the frequency response of the PLC channel. 


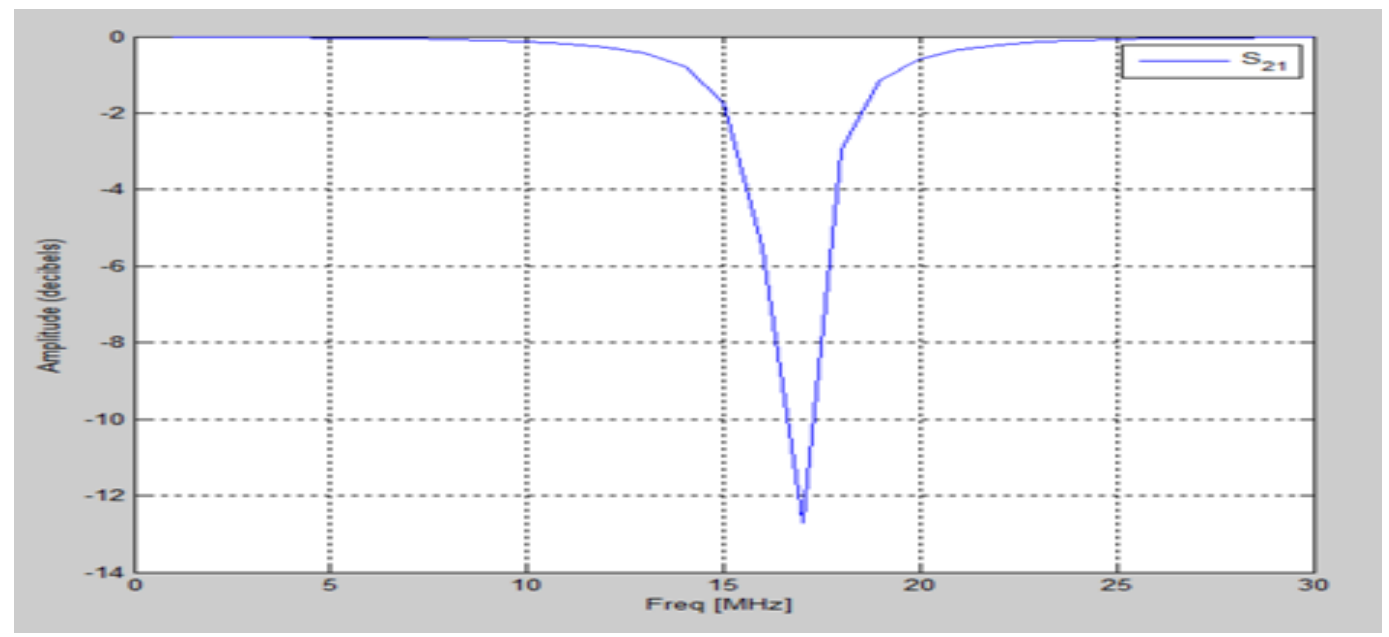

Figure 8 Amplitude spectrum of power line channel

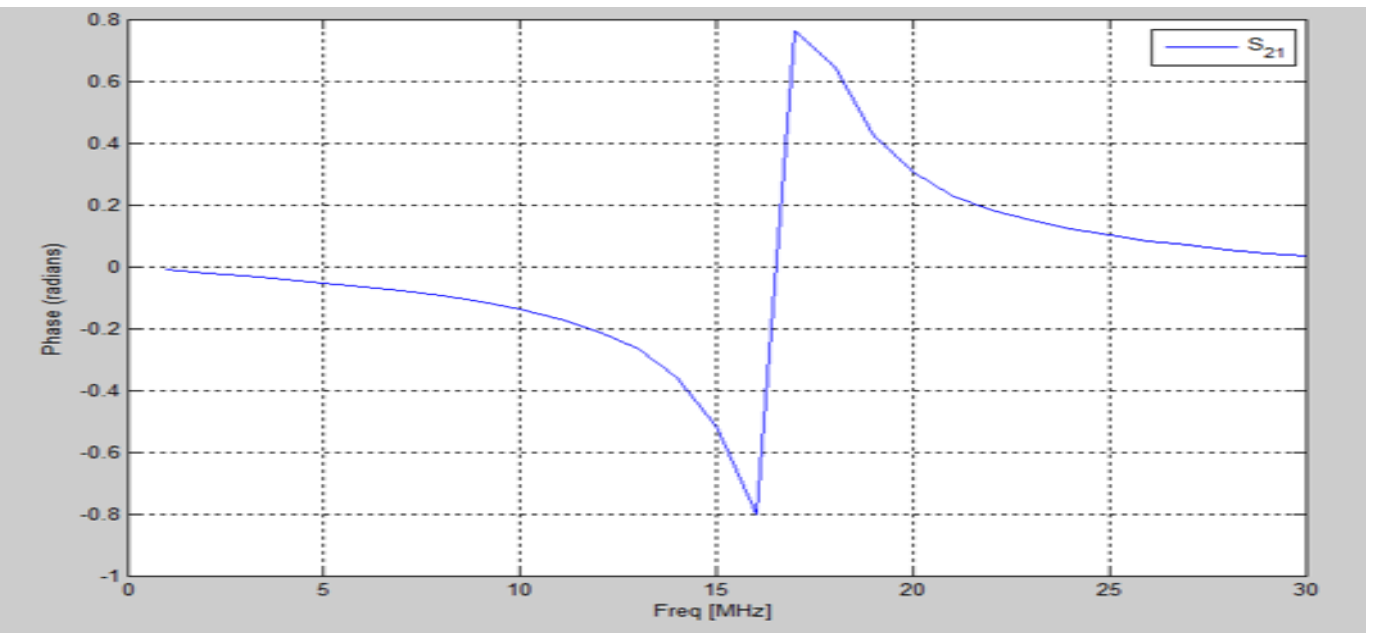

Figure 9 Phase spectrum of power line channel

\section{Performance evaluation of PLCC}

In this section describe basic idea of the model of impulse response of the multipath propagation, which means that a transmitted signal reaches the receiver on several paths with different delay and different attenuation. This model, which is called echo model, can be used to describe a certain channel by a limited set of parameters and it has been used to build up a statistical channel representation signifying different types of typical power line channels. Such models are used for performance evaluation of power line carrier communication, 100000 bits are transmitted, and bit error rate are measured at receiving end. Plot is drawn between bit error rate and signal to noise ratio ranging from 0 to $40 \mathrm{~dB}$ at a frequency $50 \mathrm{~Hz}$ and modulation BPSK respectively. Simulink debugger allows us to run a simulation model stopping the simulation results examine of executed model.
Echo due to the existence of joints or obstacles in the channel environment, the transmitted signal would travel more than just one path to arrive the receiver. As result of signals along different paths having different line attenuation, phase excursion and arriving time, the received signal is the vector superposition of all signals travelling along different paths. Then we get the echo effect. In most electric power-line connections, the attenuation of the signal increases with frequency. The frequency dependent attenuation that created deep or profound notches, these notches shown in the transfer function, which may be extending over the entire frequency range. These profound notches are caused by several reflections at impedance discontinuities. The extent of the impulse reaction and the number of the aroused peaks can vary considerably depending on the environment. Reflections at impedance discontinuities source echoes of send out signal. 
Each sent signal arrives at the receiver not only on a straight path, but also delayed and in most cases attenuated. Due to this information, an echo model has been built up, which is in good compliance with the physical parameters of the network [12], [13]. An "echo model" of the channel as illustrated in Figure 10.

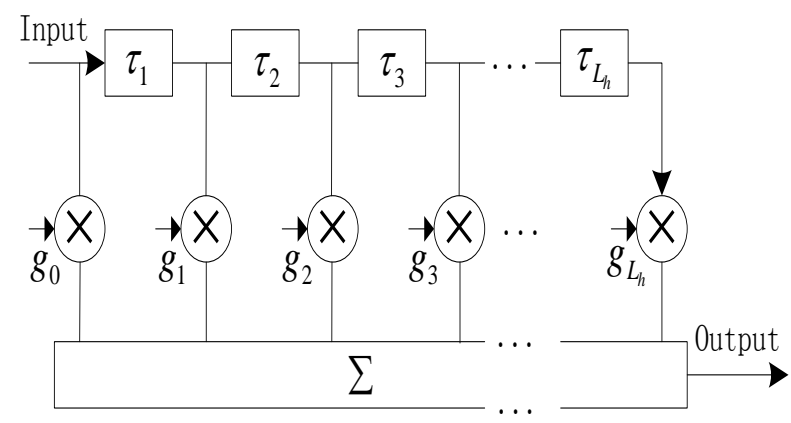

Figure 10 Basic structure of echo model

Where $g_{i}$ is the weighting factor representing the product of the reflection and transmission factors along the path $\mathrm{i}$ and $\tau_{i}$ is the path delay.

Philipp's echo model describes the channel impulse response as a superposition of $\mathrm{N}$ Dirac pulses representing the superposition of signals from $\mathrm{N}$ different paths. Each of these impulses is multiplied by a weighting factor $g_{i}$ and the delayed by $\tau_{i}$. The factor $\mathrm{g}_{\mathrm{i}}$ represent product of reflection transmission factors along each echo path. This lead to complex channel transfer function [13].

$$
h(t)=\sum_{i=1}^{N} g_{i} \delta\left(t-\tau_{i}\right)
$$

Taking into account the line attenuation due to heat loss and radiation and taking the fast Fourier transform, the channel response is given as

$$
h(f)=\sum_{i=1}^{N} g_{i} \cdot e^{-\alpha(f) d_{i}} \cdot e^{-j 2 \pi \tau_{i}}
$$

Where $g_{i}$ is the weighting factor representing the product of the reflection and transmission factors along the path i, $\tau_{i}$ is the path delay commenced by path i. After approximation, propagation constant $\gamma$ can be written as

$$
\gamma=\frac{1}{2}\left[\frac{R}{Z_{0}}+G Z_{0}\right]+j w \sqrt{L C .}
$$

The attenuation constant (i.e., the real part of $\gamma$ ) is

$$
\alpha=\frac{1}{2}\left[\frac{R}{Z_{\mathrm{o}}}+G Z_{\mathrm{o}}\right]
$$

After substituting the values of $R G$, and $Z_{0}$ the attenuation constant is approximated as

$$
\alpha k_{1} \sqrt{f}+k_{2} f
$$

Where $k_{1}$ and $k_{2}$ are constants depending on the material and dimensional characteristics of the conductors. It is the exponent of the attenuation usually in the interval of 0.2 to 1 .

$$
\tau_{i}=\frac{d_{i}}{v_{p}}
$$

$\tau_{i}$ is the path delay introduced by path $\mathrm{i}$ and is the ratio of the path length $d_{i}$ and phase velocity $v_{p} \cdot$

$$
v_{p}=\frac{\beta}{\omega}
$$

Multipath propagation approaches, which are suitable for describing the transmission behavior of power line channels, have been proposed by Philipp's [10] and Zimmermann [12].

Table 2 Parameter of power line channel

\begin{tabular}{lll}
\hline Path number & Attenuation $\mathbf{G}_{\mathbf{I}}$ & Delay $\mathbf{T}_{\mathbf{I}}$ \\
\hline 1 & 0.875 & 0 \\
2 & 0.1775 & $0.315 \mathrm{e}-6$ \\
3 & 0.07 & $0.579 \mathrm{e}-6$ \\
4 & 0.0525 & $1 \mathrm{e}-6$ \\
5 & 0.0325 & $1.3 \mathrm{e}-6$ \\
6 & 0.0325 & $1.8 \mathrm{e}-6$ \\
\hline
\end{tabular}

The dominant paths of the impulse response are sufficiently covered by the simple $\mathrm{N}=6$ path model from which the attenuations and delays are calculated to develop a six-path echo model in Simulink as shown below in Figure 11.

For verification of Simulink model for multipath channel, we transmit a signal and observed impulse response of model channel that is described below in output response of Figure 12. 
The length of the impulse response and the number of the occurred peaks can vary considerably depending on the environment. This behavior can be described by an "echo model" of the channel. Impulse response plot of Echo model is obtained after running the program [14].

The performance of power line channel communication is shown in figure with BPSK modulation at $50 \mathrm{kHz}$ over the SNR ranging 0 to 40 $\mathrm{dB}$ and no of bits 10000 .

The display block showed the following bit error rate as shown in Figure 14 obtained after running the model i.e. when both channel delay and the effect of attenuation is considered under the effect of both delay and attenuation as shown in Figure 14. We observed that increasing the value of SNR, BER decreasing regularly [15].

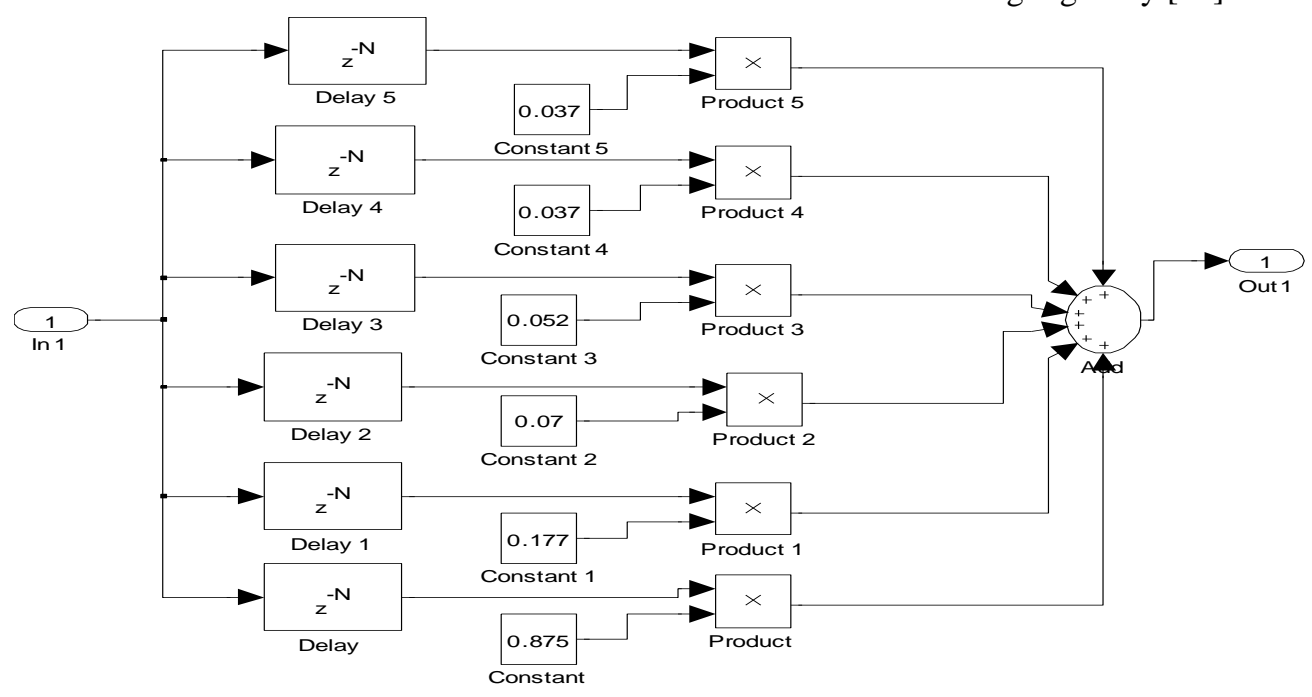

Figure 11 Simulink model for multipath channel
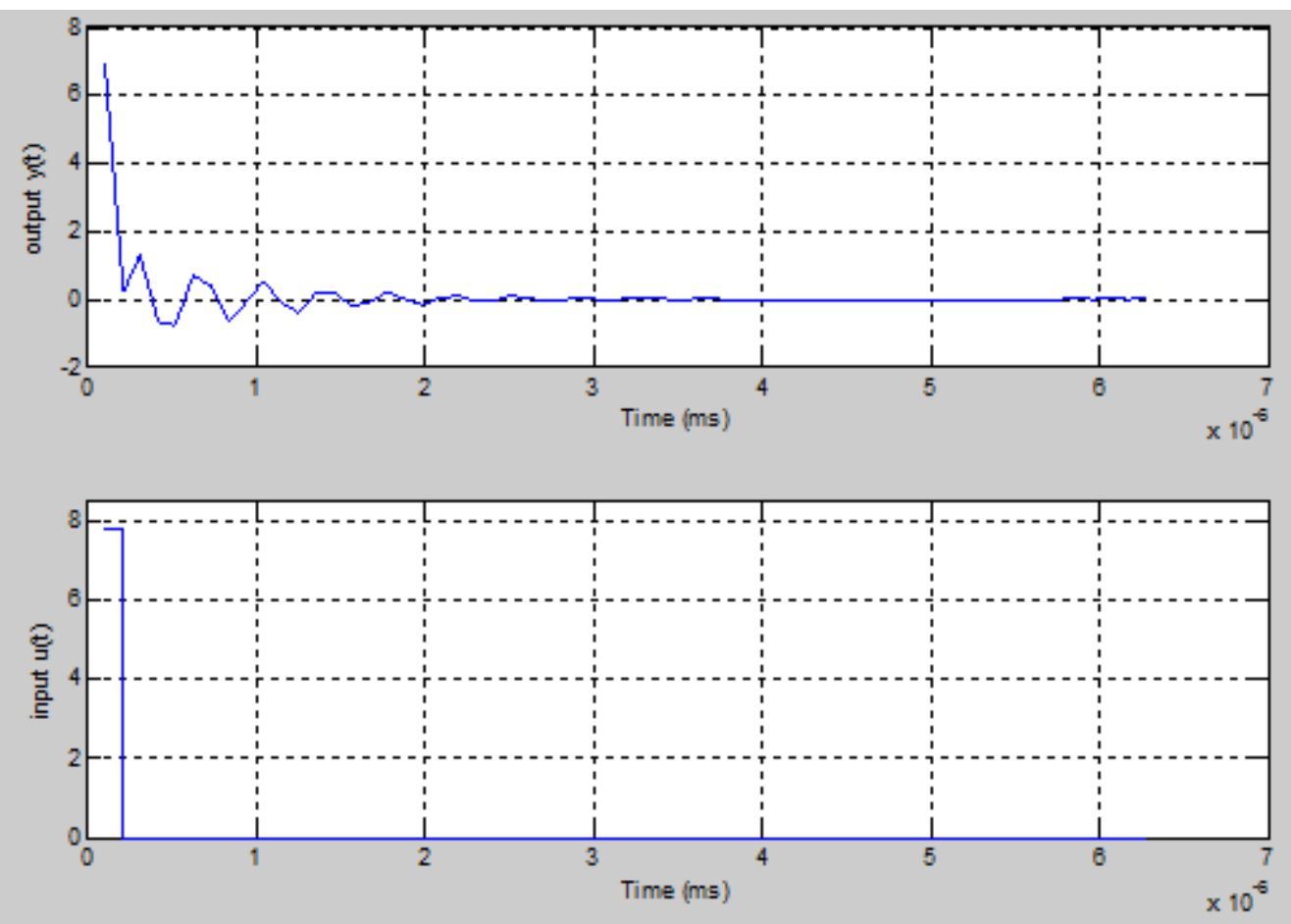

Figure 12 Impulse response of multipath channel 


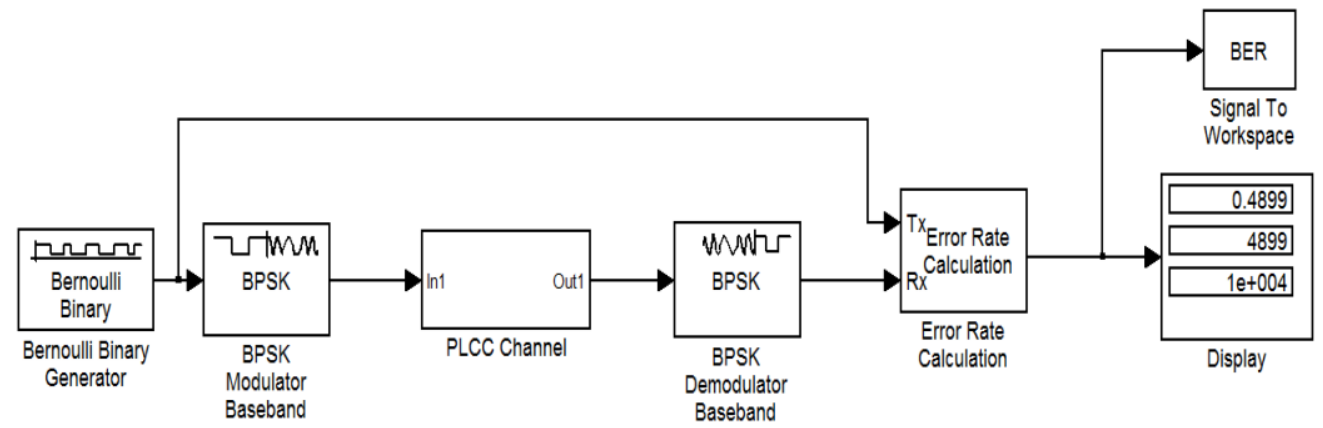

Figure 13 Performance evaluation of modelled channel

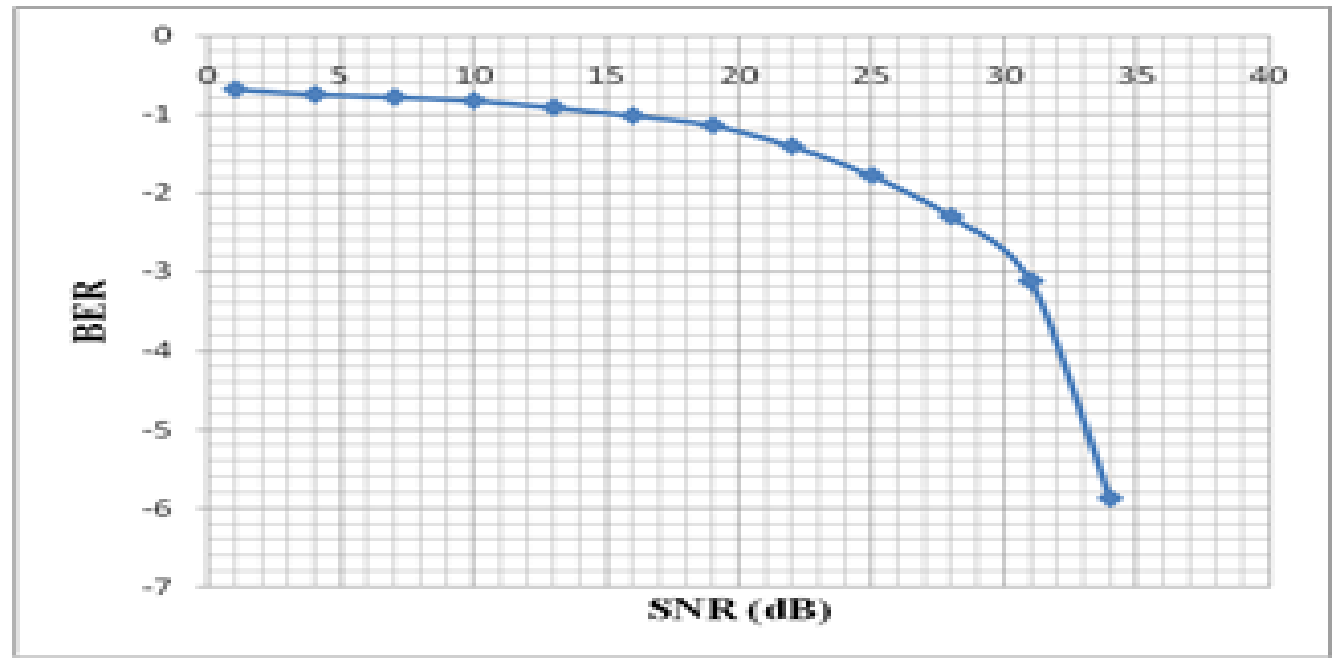

Figure 14 SNR v/s BER Plot for PLCC channel

\section{Conclusion}

The low voltage communication channel is modeled as two wire transmission lines and use their intrinsic parameters namely characteristic impedance, propagation constant that is used to derived other parameters, for the simulation of transfer function for PLCC channel which is determined based on twoport network theory. The two-port network is represented with the reflected and incident matrix theory also known as the scattering matrix theory. Based on the simulation result, the property of the power line channel is found that the channel attenuation increases with the increase in frequency. This paper describes the basic idea of the model of impulse response of the multipath transmission, that the sent-out signal which reaches at receiver on several paths with unusual delay and attenuation that representation is known as echo model, which is employed to express an assured channel by a limited set of parameters. Such models are used for performance evaluation of power line carrier communication. 100000 bits are transmitted, and bit 316 error rate are measured at receiving end. These results are of great importance in determining the attenuation and delay of PLCC. As the data rate depends upon the physical parameters of PLCC, the maximum reliable data rate can also be estimated using this model.

There are a number of areas in this paper that can be extended through further research using this modeling concept, these are few such as channel and noise measurements, study and effect of differences in topologies, effect of structures and types of wire, loading effect in Indian grids, last and most important future research is mitigating the problems using new technology.

\section{Acknowledgment}

None.

\section{Conflicts of interest}

The authors have no conflicts of interest to declare. 


\section{References}

[1] Hossain E, Khan S, Ali A. Low voltage power line characterization as a data transfer method in public electricity distribution networks and indoor distribution networks. In electric power conference 2008 (pp. 1-7). IEEE.

[2] Ferreira HC, Grove HM, Hooijen O, Vinck AH. Power line communications: an overview. In AFRICON 1996 (pp. 558-63). IEEE.

[3] Meng H, Chen S, Guan YL, Law CL, So PL, Gunawan E, et al. Modeling of transfer characteristics for the broadband power line communication channel. IEEE Transactions on Power Delivery. 2004; 19(3):1057-64.

[4] Galli S, Scaglione A, Wang Z. For the grid and through the grid: the role of power line communications in the smart grid. Proceedings of the IEEE. 2011; 99(6):998-1027.

[5] Berger LT, Iniewski K. Smart grid: applications, communications, and security. Wiley; 2012.

[6] Meng H, Chen S, Guan YL, Law CL, So PL, Gunawan E, et al. A transmission line model for highfrequency power line communication channel. In power system technology, proceedings international conference on 2002 (pp. 1290-5). IEEE.

[7] Paul CR. Analysis of multiconductor transmission lines. John Wiley \& Sons; 2008.

[8] Anatory J, Kissaka MM, Mvungi NH. Channel model for broadband power-line communication. IEEE Transactions on Power Delivery. 2007; 22(1):135-41.

[9] Galli S, Banwell T. A novel approach to the modeling of the indoor power line channel-part II: transfer function and its properties. IEEE Transactions on Power Delivery. 2005; 20(3):1869-78.

[10] Philipps H. Modelling of powerline communication channels. In proceedings of the symposium of power line communications for smart grid applications 1999 (pp. 14-21).

[11] Berger LT, Moreno-Rodriguez G. Power line communication channel modelling through concatenated IIR-filter elements. Journal of Communications. 2009; 4(1):41-51.

[12] Zimmermann M, Dostert K. A multipath model for the powerline channel. IEEE Transactions on Communications. 2002; 50(4):553-9.
[13] Konate C, Machmoum M, Diouris JF. Multi path model for power line communication channel in the frequency range of $1 \mathrm{mhz}-30 \mathrm{mhz}$. In the international conference on" computer as a tool" 2007 (pp. 984-9). IEEE.

[14] Tonello AM, Versolatto F. Bottom-up statistical PLC channel modeling-part I: random topology model and efficient transfer function computation. IEEE Transactions on Power Delivery. 2011; 26(2):891-8.

[15] Chandna VK, Zahida M. Effect of varying topologies on the performance of broadband over power line. In transmission and distribution conference and exposition 2010 (pp. 1-5). IEEE.

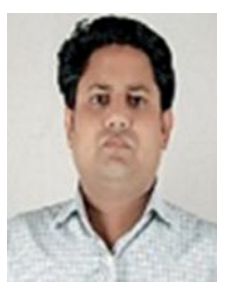

Mr. Zahoor Alam has done his postgraduation in Engineering from Jamia Millia Islamia, New Delhi. $\mathrm{He}$ is currently working as Industrial Trainer at Amity Education Services Pvt Ltd., Bikaner (Rajasthan). His research area is Power System Automation, Integration of Renewable Energy into the Grid and Energy Management.

Email: zahooralam10@gmail.com

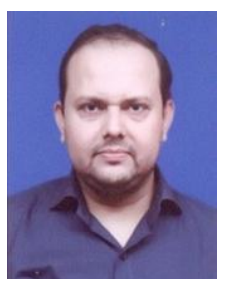

Mr. Aizad Khursheed has done his graduation and post-graduation in Engineering from Jamia Millia Islamia, New Delhi. He is currently working as Assistant Professor at Amity University, Greater Noida. His research area is Power System Operation and Control (Microgrid) and Power Quality.

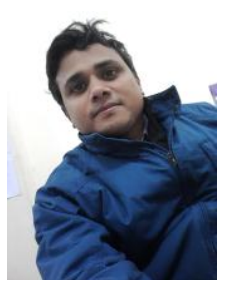

Mr. Rahul Kant Chaudhary has done his post-graduation in Engineering from Jamia Millia Islamia, New Delhi. His research area is Power System Automation and Integration of Renewable Energy into the Grid. 